\title{
Monoarthropathy or Polyarthritis in Adolescent Japanese Girls Who Received Immunization with the Human Papillomavirus Vaccine
}

\author{
Ryuta Abe, Tomomi Kinoshita, Akiyo Hineno, Shu-ichi Ikeda* \\ Department of Medicine (Neurology and Rheumatology), Shinshu University School of Medicine, \\ Matsumoto, Japan \\ Email: ${ }^{*} i k e d a s i @ s h i n s h u-u . a c . j p$
}

Received 19 February 2016; accepted 20 March 2016; published 23 March 2016

Copyright (C) 2016 by authors and Scientific Research Publishing Inc.

This work is licensed under the Creative Commons Attribution International License (CC BY). http://creativecommons.org/licenses/by/4.0/

(c) (i) Open Access

\begin{abstract}
Joint pain or arthralgia is a common complaint among girls who have received immunization with the human papillomavirus (HPV) vaccine, but the pathogenesis of this disorder has not been completely understood. We report 2 cases of joint lesions after HPV vaccination. In one case, a 13-yearold patient showed transient arthropathy in the right wrist joint after the first dose of Gardasil ${ }^{\circledR}$ administered in her left shoulder. In the other case, an 18-year-old patient had migrating joint pain with redness and swelling after the third dose of Cervarix ${ }^{\circledR}$. Her serum C-reactive protein and anti-MMP-3 levels were slightly elevated, but no autoantibodies, including rheumatoid factor and anti-CCP antibody, were detected. Although various nonsteroidal anti-inflammatory drugs produced no relief, small doses of both tacrolimus and prednisolone were highly effective for her polyarthritis. The development of reactive joint lesions after HPV vaccination was noteworthy.
\end{abstract}

\section{Keywords}

Human Papillomavirus Vaccine, Adverse Reaction, Arthritis, Arthralgia, Juvenile Idiopathic Arthritis

\section{Introduction}

Immunization of adolescent girls with the human papillomavirus (HPV) vaccine was initiated to prevent uterine

${ }^{*}$ Corresponding author.

How to cite this paper: Abe, R., Kinoshita, T., Hineno, A. and Ikeda, S. (2016) Monoarthropathy or Polyarthritis in Adolescent Japanese Girls Who Received Immunization with the Human Papillomavirus Vaccine. Case Reports in Clinical Medicine, 5, 109-114. http://dx.doi.org/10.4236/crcm.2016.53020 
and cervical cancers [1] [2]. In April 2013, two types of vaccines (Gardasil ${ }^{\circledR}$-CSL/Merck and Cervarix ${ }^{\circledR}$-GSK) were widely introduced for use in Japanese girls aged 13 to 16 years. The adverse events that occur after HPV vaccination commonly include fever, headache, and local pain at the injection site [3]. In addition, a relatively high incidence of chronic limb pain, frequently complicated by violent, tremulous involuntary movements, has been noted in Japanese girls after receiving vaccination.

Our previous report showed that the main disorders observed in these post-vaccination girls consisted of complex regional pain syndrome (CRPS)I, orthostatic hypotension (OH), and postural orthostatic tachycardia syndrome (POTS), all of which were ascribed to peripheral sympathetic nerve dysfunction [4]. Similar data were recently reported from Denmark [5], and for the pathophysiology of this dysautonomia, small fiber neuropathy is surmised to be an underlying disorder [6]. Migrating joint pains or arthralgias were frequent systemic reactions to HPV vaccination [7], but usually lacked any inflammatory findings, including elevated serum C-reactive protein (CRP) levels. These joint pains were therefore interpreted as a manifestation of CRPS-I [8] [9]. We here describe two Japanese patients who developed true joint lesions after HPV vaccination.

\section{Case Report}

\subsection{Case 1}

In June 2013, a 13-year-old girl received her first dose of Gardasil ${ }^{\circledR}$ in the left shoulder. The next evening, she felt pain in the right wrist joint, which lasted for a few days. The pain was markedly induced by flexion of the joint, and cold packing was not effective. One week later, she visited her previous physician, but no abnormality was found on the roentgenogram of the joint. She was then referred to us. On the 10th post-injection day, she had neither fever nor lymphadenopathy, but slight tenderness was present in her right wrist joint. Laboratory examination results showed a serum CRP level of $0.12 \mathrm{mg} / \mathrm{dl}$ and were negative for autoantibodies, including rheumatoid factor and anti-cyclic citrullinated peptide (CCP) antibody. Magnetic resonance imaging (MRI) revealedslight fluid accumulation in her right wrist joint (Figure 1), without signal change on articular soft tissues or bones. She was therefore diagnosed as having mono-arthropathy. As she had no difficulty in using the right hand, no medication was prescribed, and the joint pain spontaneously subsided over the following few days. Further vaccinations were not administered.

\subsection{Case 2}

In August 2014, an 18-year-old girl was referred to our hospital because of polyarthralgia. She received the first dose of Cervarix ${ }^{\circledR}$ at the end of February 2012 and the third dose at the end of August in the same year. Soon after the final vaccination, she started to experience arthralgia in the left knee joint and visited an orthopedic clinic, where she was diagnosed as having physical stress due to playing basketball and underwent plicotomy under

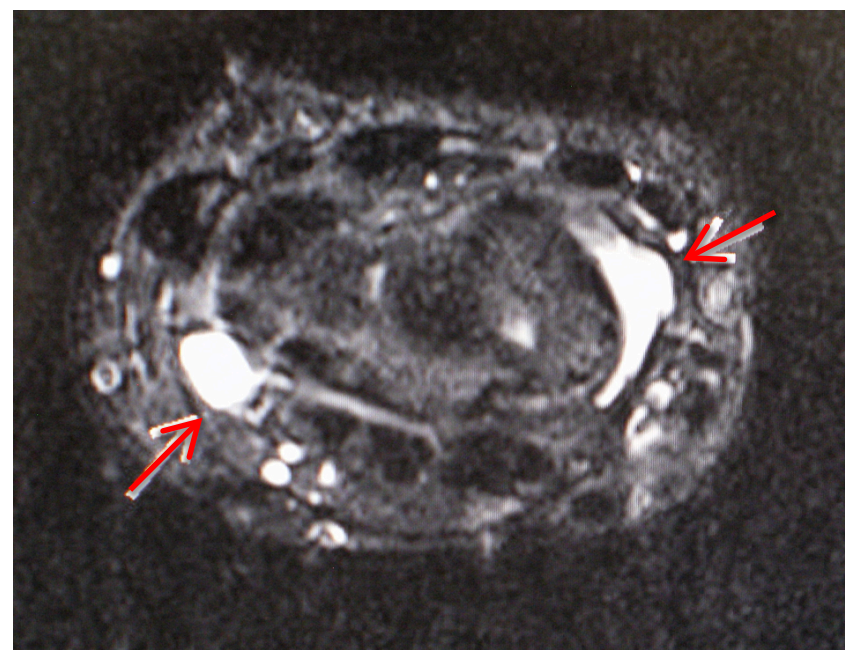

Figure 1. MRI finding of the right wrist joint in case 1. Short T1 inversion recovery (STIR) image revealing a small amount of synovial fluid accumulation indicated by arrows. 
endoscopic control. However, the left knee joint pain continued, and arthralgia appeared in the right knee joint. However, pain in both joints subsided over the next 2 weeks without any treatments. During the following 6 months, arthralgia with joint swelling appeared and spread around the elbows, wrists, and ankle joints. In addition, she had intermittent pyrexia (body temperature, $37^{\circ} \mathrm{C}-38^{\circ} \mathrm{C}$ ). In February 2013 , she visited the department of orthopedics of another hospital because of left hand swelling and pain (Figure 2(a)). The roentgenograms of the elbow, wrist, knee and ankle joints were unremarkable. Rheumatoid factor was not detected in serum samples, but the serum CRP and anti-matrix metalloproteinase (MMP) levels were $2.22 \mathrm{mg} / \mathrm{dl}$ and $73.7 \mathrm{ng} / \mathrm{ml}$ (normal: 17.3 - 59.7), respectively. The erythrocyte sedimentation rate was elevated (45 mm/1 hour). She was suspected to have juvenile idiopathic arthritis (JIA) and was administered different types of nonsteroidal anti-inflammatory drugs (NSAIDs), which provided no relief. She was then referred to our hospital.

On examination, she had no fever and lacked any physical or neurological findings except for polyarthropathy. Slight to moderate tenderness was observed in the shoulder, elbow, wrist, and knee joints, and both knee joints showed mild, fluctuating swelling. Her serum CRP level was $0.21 \mathrm{mg} / \mathrm{dl}$. No autoantibodies, including anti-CCP antibody, anti-nuclear antibody, and anti-double-strand DNA, were detected. We found no evidence suggesting a previous viral exposure, including Epstein-Barr virus or human parvovirus B19. HLA genotyping performed in a commercially available laboratory disclosed the following findings: DPB1: *02:01:02/*14:01, DQA1: *03:03/*04:01:01, DQB1: *04:01:01/*04:02:01, and DRB1: *04:05/*16:02. MRI revealed moderate fluid accumulation in both knee joints (Figure 3). Accordingly, she was tentatively diagnosed as having reactive arthri-tis after HPV vaccination. She was again administered celecoxib 200mg/day; but 6 months later, she deve-
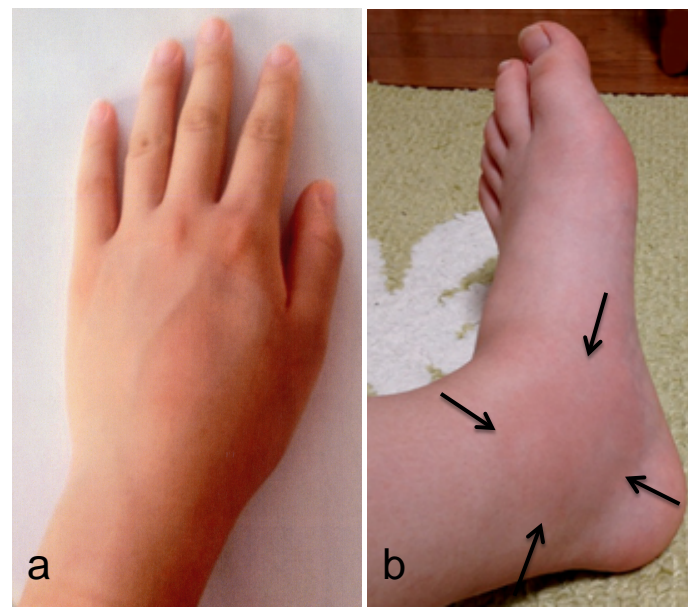

Figure 2. Arthritis finding in case 2. (a) The left hand showing swelling with redness; (b) The left ankle joint showing swelling with redness (indicated by arrows).

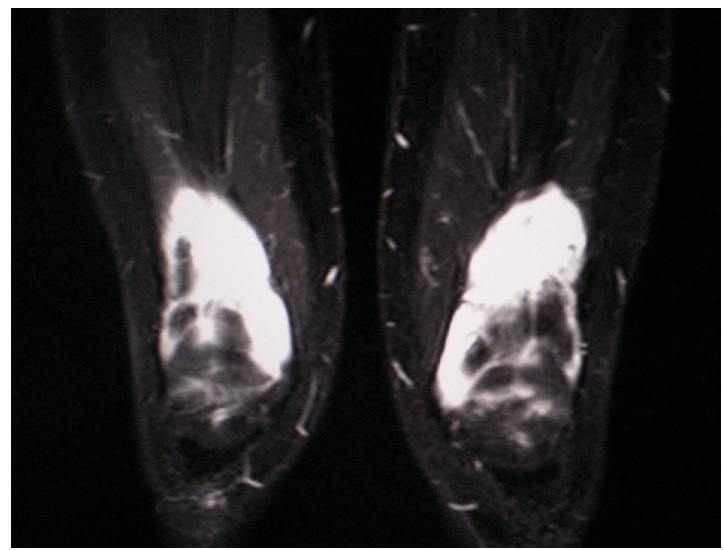

Figure 3. MRI finding of the knee joints in case 2. STIR image showing amoderate amount of synovial fluid accumulation. 
loped marked swelling and redness with pain in the left ankle joint (Figure 2(b)). This caused difficulty in going up or down stairs. Her serum CRP and anti-MMP-3 levels were elevated to $2.12 \mathrm{mg} / \mathrm{dl}$ and $109.7 \mathrm{ng} / \mathrm{ml}$, respectively. Her medication was therefore switched to daily doses of tacrolimus $(1.5 \mathrm{mg})$ and prednisolone $(10 \mathrm{mg})$, the latter being gradually tapered to $7 \mathrm{mg}$ per day over the next 8 months. Methotrexate was not chosen as therapeutic agent, as she had much anxiety regarding its side effect. As a result, her polyarthritis did not recur, and she has since been spending normal school life for 15 months.

\section{Discussion}

In Japan, 8.75 million doses of HPV vaccine, predominantly Cervarix ${ }^{\circledR}$, have been administered during the past 3 years. Between December 2009 and March 2013, 698 cases were reported to have had serious adverse reactions. Among these reactions, syncope, transient unconsciousness, anaphylactic shock, severe limb pain with paresis, and/or involuntary movement were the most frequent manifestations. Eleven cases with arthritis have been reported, in which all of the patients received Cervarix ${ }^{\circledR}$ (incidence of arthritis: 0.016\%), but the details of these joint lesions were not reported (information from the Ministry of Public Health, Labour and Welfare, Japan; see http://www.mhlw.go.jp/stf/shingi/0000033881.html). Arthritis is known to be a post-vaccination phenomenon [10]. Diphtheria-tetanus-pertussis (DTP) and measles-mumps-rubella (MMR) vaccines are representative causative agents. Aberrant autoimmune responses after vaccination are surmised to be responsible for this form of arthritis [11]. Experimental animals immunized by vaccines were found to produce several different autoantibodies, including lupus-associated ones [12]. Some adjuvants such as aluminium salts may play an important role in the pathogenesis of these immune mediated disorders [13] [14]. Recently, the new term autoimmune (autoinflammatory) syndrome induced by adjuvants (ASIA) has been proposed to cover four similar conditions consisting of silicosis, the Gulf War syndrome, the macrophagic myofasciitis syndrome, and post-vaccination phenomena [10] [15]. In the diagnostic criteria for ASIA, arthralgia and/or arthritis is listed as a typical manifestation [10]. The presence of some HLA types such as HLA-DRB1 or DQB1 seems to be a diagnostic hallmark for ASIA [10] [16]. In addition, in a small number of Japanese girls, the DPB1*05:01 allele was reported to be significantly associated with the post-HPV vaccinated neurological disorder [17]. The epidemiological characteristic of ASIA among the girls who received HPV vaccination was evaluated based on the Vaccine Adverse Reporting System (VAERS). The estimated reporting rate was 3.6 cases per 100,000 doses, and the significant odds ratio of developing SLE, vasculitis, and arthritis were 5.3 (1.5 - 20.5), 4 (1.01 - 16.4), and 2.5 (1.4 - 4.3), respectively [18].

In our two cases, joint lesions developed soon after HPV vaccination, suggesting that the disorders were causally related to HPV vaccination. Case 1 showed transient mono-arthropathy without any specific treatment, while case 2 followed a pattern of recurrent polyarthritis and finally required immunomodulating therapy. Thus, in the latter case, the clinical picture and treatment response closely resembled those of JIA [19], although the patient lacked destructive joint lesions or systemic involvement of visceral organs. In the Japanese reports of adverse events after HPV vaccination, the incidence of arthritis was low. Thus, some physicians insist that JIA independently developed in these girls [3]. This possibility cannot be certainly ruled out, as JIA or rheumatoid arthritis is one of the most common forms of autoimmune arthritis, affecting approximately $0.5 \%-1.0 \%$ of the general population [20]. In contrast, no specific diagnosis for untoward vaccine reactions has been established. The HLA genotype obtained from case 2 seemed not determinative of the final diagnosis. We therefore surmise that a significant number of cases with this disorder were misdiagnosed as having CRPS-I, fibromyalgia, or psychogenic pain [21]. The pathogenic mechanism of the occurrence of serious adverse reactions, including dysautonomia, after HPV vaccination remains unclear [6]. Nevertheless, to respond practically, clinicians should be aware of the possible association between HPV vaccination and the development of arthropathy or arthritis. To our knowledge, this is the first report describing the details of joint lesions that developed after HPV vaccination.

\section{Conclusion}

HPV vaccination may occasionally induce true joint lesions.

\section{Acknowledgements}

This work was supported by a Health and Labour Science Research Grant on Intractable Diseases (Pathogenesis 
and Diagnostic Accuracy of Neuropathic Pain, 23170301 to S.I.) from the Ministry of Public Health, Labour and Welfare, Japan.

\section{Conflict of Interest}

None.

\section{References}

[1] The Future II Study Group (2007) Quadrivalent Vaccine against Human Papillomavirus to Prevent High-Grade Cervical Lesions. New England Journal of Medicine, 356, 1915-1927. http://www.nejm.org/doi/full/10.1056/NEJMoa061741

[2] Jeurissen, S. and Makar, A. (2009) Epidemiological and Economic Impact of Human Papillomavirus Vaccines. International Journal of Gynecological Cancer, 19, 761-771. http://dx.doi.org/10.1111/IGC.0b013e3181a130c0

[3] Slade, B.A., Leidel, L., Vellozzi, C., et al. (2009) Postlicensure Safety Surveillance for Quadrivalent Human Papillomavirus Recombinant Vaccine. JAMA, 302, 750-757. http://dx.doi.org/10.1001/jama.2009.1201

[4] Kinoshita, T., Abe, R., Hineno, A., Tsunekawa, K., Nakane, S. and Ikeda, S. (2014) Peripheral Sympathetic Nerve Dysfunction in Adolescent Japanese Girls Following Immunization with the Human Papillomavirus Vaccine. Internal Medicine, 53, 2185-2200. http://dx.doi.org/10.2169/internalmedicine.53.3133

[5] Brinth, L., Theibel, A.C., Pors, K. and Mehlsen, J. (2015) Suspected Side Effects to the Quadrivalent Human Papilloma Vaccine. Danish Medical Journal, 62, A5064.

[6] Martínes-Lavín, M. (2015) Hypothesis: Human Papillomavirus Vaccination Syndrome-Small Fiber Neuropathy and Dysautonomia Could Be Its Underlying Pathogenesis. Clinical Rheumatology, 34, 1165-1169. http://dx.doi.org/10.1007/s10067-015-2969-z

[7] Gasparini, R., Bonanni, P., Levi, M., et al. (2011) Safety and Tolerability of Bivalent HPV Vaccine: An Italian PostLicensure Study. Human Vaccines, 7, 136-146. http://dx.doi.org/10.4161/hv.7.0.14576

[8] Bruehl, S., Harden, R.N., Galer, B.S., et al. (1999) External Validation of IASP Diagnostic Criteria for Complex Regional Pain Syndrome and Proposed Research Diagnostic Criteria. Pain, 81, 147-154. http://www.sciencedirect.com/science/article/pii/S0304395999000111 http://dx.doi.org/10.1016/S0304-3959(99)00011-1

[9] Richards, S., Chalkiadis, G., Lakshman, R., Buttery, J.P. and Crawford, N.W. (2012) Complex Regional Pain Syndrome Following Immunisation. Archives of Disease in Childhood, 97, 913-915. http://dx.doi.org/10.1136/archdischild-2011-301307

[10] Shoenfeld, Y. and Agmon-Levin, N. (2011) “ASIA”-Autoimmune/Inflammatory Syndrome Induced by Adjuvants. Journal of Autoimmunity, 36, 4-8. http://dx.doi.org/10.1016/j.jaut.2010.07.003

[11] Agmon-Levin, N., Paz, Z., Israeli, E. and Shoenfeld, Y. (2009) Vaccines and Autoimmunity. Nature Reviews Rheumatology, 5, 648-652. http://dx.doi.org/10.1038/nrrheum.2009.196

[12] Hogenesch, H., Azcona-Olivera, J., Scott-Moncrieff, C., Snyder, P.W. and Glickman, L.T. (1999) Vaccines Induced Autoimmunity in Dogs. Advances in Veterinary Medicine, 41, 733-747. http://dx.doi.org/10.1016/S0065-3519(99)80056-1

[13] Gherardi, R.K., Coquet, M., Chérin, P., et al. (1998) Macrophagic Myofasciitis: An Emerging Entity. Groupe d’Etudes et Recherche sur les Maladies Musculaires Acquises et Dysimmunitaires (GERMMAD) de l'Association Française contre les Myopathies (AFM). Lancet, 352, 347-352. http://dx.doi.org/10.1016/S0140-6736(98)02326-5

[14] Authier, F.J., Cherin, P., Creange, A., et al. (2001) Central Nervous System Disease in Patients with Macrophagic Myofasciitis. Brain, 124, 974-983. http://dx.doi.org/10.1093/brain/124.5.974

[15] Perricone, C., Colafrancesco, S., Mazor, R.D., Soriano, A., Agmon-Levin, N. and Shoenfeld, Y. (2013) Autoimmune/Inflammatory Syndrome Induced by Adjuvants (ASIA) 2013: Unveiling the Pathogenic, Clinical and Diagnostic Aspects. Journal of Autoimmunity, 47, 1-16. http://dx.doi.org/10.1016/j.jaut.2013.10.004

[16] Anaya, J.M., Reyes, B., Perdomo-Arciniegas, A.M., Camacho-Rodríguez, B. and Rojas-Villarraga, A. (2015) Autoiimune/Autoinflammatory Syndrome Induced by Adjuvants (ASIA) after Quadrivalent Human Papillomavirus Vaccination in Colombians: A Call for Personalized Medicine. Clinical and Experimental Rheumatology, 33, 545-548.

[17] Arata, H., Ando, M., Okada, T., et al. (2015) Studies of Encephalopathy Associated with ASIA after HPV Vaccination. Clinical Neurology, 55, S52.

[18] Pellegrino, P., Perrone, V., Pozzi, M., et al. (2015) The Epidemiological Profile of ASIA Syndrome after HPV Vaccination: An Evaluation Based on the Vaccine Adverse Event Reporting Systems. Immunologic Research, 61, 90-96. 
http://dx.doi.org/10.1007/s12026-014-8567-3

[19] Yokota, S., Mori, M., Imagawa, T., et al. (2007) Proposal for Juvenile Idiopathic Arthritis Guidance on Diagnosis and Treatment for Primary Care Pediatricians and Nonpediatric Rheumatologists. Modern Rheumatology, 17, 353-363. http://dx.doi.org/10.3109/s10165-007-0625-4

[20] Kochi, Y., Suzuki, A. and Yamamoto, K. (2014) Genetic Basis of Rheumatoid Arthritis: A Current Review. Biochemical and Biophysical Research Communications, 452, 254-262. http://dx.doi.org/10.1016/j.bbrc.2014.07.085

[21] Buttery, J.P., Madin, S., Crawford, N.W., et al. (2008) Mass Psychogenic Response to Human Papillomavirus Vaccination. Medical Journal of Australia, 189, 261-262. 